\title{
Traducción y adaptación transcultural del cuestionario para niños «Barriers and facilitators of sports in children with physical disabilities (BaFSCH)» para su uso al español en Chile Transcultural translation and adaptation of the questionnaire for children «Barriers and facilitators of sports in children with physical disabilities (BaFSCH)» for use into Spanish in Chile
}

*Fernando Ignacio Muñoz Hinrichsen, **Alan Frederik Martínez Aros, ***Felipe Herrera Miranda *Universidad Metropolitana de Ciencias de la Educación (Chile), **Universidad Andres Bello (Chile), ***Universidad Viña del Mar (Chile)

Resumen. El objetivo de este estudio fue realizar una adecuación transcultural del cuestionario «Barriers and facilitators of sports in children with physical disabilities» al idioma español hablado en Chile. Para el proceso de traducción y adaptación transcultural se siguieron los pasos de traducción directa, síntesis de traducciones, traducción inversa, consolidación de comité de expertos y pre-test. En cuanto a los resultados se realizaron los pasos según la metodología desarrollando las adaptaciones necesarias que se consideraron desde la opinión de los expertos y traductores que participaron de la adaptación del escrito. Participaron 93 sujetos con un promedio de edad de 13,95 años $\pm 4,84$ donde hubo 42 niñas $(45,16 \%)$ y 51 niños $(54,84 \%)$. La mayoría de las y los participantes asisten a educación básica $(62,36 \%)$, con condiciones no definidas $(34,40 \%)$ seguido de quienes tiene dificultades para movilizarse (12,90\%) y quienes no comprenden bien las indicaciones $(12,90 \%)$. Se considera que este cuestionario puede ser una herramienta adecuada para conocer la percepción de los participantes lo que puede finalmente impactar en diversas acciones y políticas que permitan la participación efectiva en actividad física y deporte bajo un enfoque de derecho social.

Palabras Claves: Niños, Discapacidad, Inclusión, Actividad Física y Deporte.

\begin{abstract}
The objective of this study was to carry out a cross-cultural adaptation of the questionnaire «Barriers and facilitators of sports in children with physical disabilities» into the Spanish language spoken in Chile. For the translation and crosscultural adaptation process, the steps of direct translation, translation synthesis, reverse translation, consolidation of the expert committee and pre-test were followed. Regarding the results, the steps were carried out according to the methodology developing the necessary adaptations that were considered from the opinion of the experts and translators who participated in the adaptation of the writing. 93 subjects participated with an average age of 13,95 $\pm 4,84$ years, where there were 42 girls $(45,16 \%)$ and 51 boys $(54,84 \%)$. Most of the participants attend basic education $(62,36 \%)$, with undefined conditions $(34,40 \%)$ followed by those who have difficulties to move around $(12,90 \%)$ and those who do not understand the indications well $(12,90 \%)$. It is considered that this questionnaire can be an adequate tool to know the perception of the participants, which can ultimately impact on various actions and policies that allow effective participation in physical activity and sport under a social law approach.
\end{abstract}

Key Words: Childrens, Disability, Inclusion, Physical Activity and Sports.

\section{Introducción}

La participación en actividad física y deporte corresponde a un factor importante del desarrollo social, ya que permite la promoción de la tolerancia y el respeto, contribuye al empoderamiento de las comunidades, y aporta a los objetivos de salud, educación e inclusión social (Organización de Naciones Unidades para la Educación, la Ciencia y la Cultura, 2015; Organización de Naciones Unidas, 2006, 2015), por lo tanto es fundamental conocer las barreras y los facilitadores que exis-

Fecha recepción: 28-09-21. Fecha de aceptación: 14-12-21

Fernando Ignacio Muñoz Hinrichsen

fernandoimh@gmail.com ten con el fin de abordarlas desde las brechas que estas generan (Jaarsma et al., 2015; Muñoz Hinrichsen et al., 2020) y así trabajar desde las políticas con el propósito de transformarlas en aporte a los determinantes sociales relacionados a las personas con discapacidad (PCD en adelante)(Tamayo et al., 2018).

Desde la perspectiva anterior la inclusión de niñas, niños y adolescentes con discapacidad (NNACD) es importante ya que se relaciona con convenciones internacionales como la de los derechos de los niños que propone dentro de sus artículos la participación en el juego, la actividad física y junto a esto la inclusión y no discriminación (Fondo de las Naciones Unidas para la Infancia, 1989), y la convención de los derechos de las PCD que en su artículo 30 propone que NNACD ten- 
gan las mismas oportunidades de participación y de acceso a la actividad física y en el deporte en la sociedad (Organización de Naciones Unidas, 2006). En Chile un grupo de leyes se alinea desde este prisma donde se busca el resguardo de las oportunidades y derechos de NNACD teniendo como base la Ley 20.422 (Ministerio de Desarrollo Social, 2010) y que se relaciona a la actividad física y el deporte con la Ley 20.978 que reconoce la práctica adaptada e inclusiva para NNACD (Ministerio del Deporte, 2015a), y la Ley 20.845 de la educación inclusiva con su decreto 83 que busca la diversificación de las actividades curriculares para NNACD donde la asignatura educación física es parte del desarrollo integral (Ministerio de Educación, 2015), en Chile se observa que las bases curriculares educativas están orientadas a la homogeneidad del alumnado y presenta poca o nula utilidad en el abordaje de la educación física y la salud propiamente tal en NNACD (Castillo-Retamal et al., 2021a).

El uso de cuestionarios para conocer la inclusión en la práctica de la actividad física se ha ido desarrollando fuertemente a nivel internacional con el objetivo de conocer esta temática, donde existen instrumentos para conocer las actitudes de los actores de la comunidad como el «Cuestionario de actitudes hacia la inclusión de estudiantes con discapacidad en educación física (AISDPE)» (Reina et al., 2016), el cuestionario «Structure, validity and reliability of the Children's AttitudesTowards Integrated Physical Education-Spanish version (CAIPE-SP)» (Cordente-Mesas et al., 2016), y «Actitudes de los alumnos hacia la integración en educación física (CAIPE-R)» (Ocete calvo et al., 2017). Sin embargo, conocer desde la mirada de NNACD es importante ya que desde la perspectiva ecológica es relevante la opinión de todos los componentes de la comunidad y en este caso son quienes requieren de un proceso de adaptación o inclusión para garantizar su participación efectiva (Hutzler \& Sherrill, 2007).

El Cuestionario para niños «Barriers and facilitators of sports in children with physical disabilities» (BaFSCH) (Jaarsma et al., 2015) fue adaptado de un cuestionario autoconstruido para atletas paralímpicos publicado previamente (Jaarsma et al., 2014), y contenía preguntas sobre participación deportiva. Los ítems sobre barreras y facilitadores se dividieron en factores personales y ambientales, según la Clasificación Internacional de Funcionamiento Discapacidad y Salud (Organización Mundial de la Salud, 2001) que observa la relación entre las estructuras corporales y el funcionamiento, las actividades y la participación social, y su relación los factores personales y del entorno. Los elementos sobre la participación deportiva y las discapacidades se agruparon de acuerdo con los componentes de la teoría del comportamiento planificado (Ajzen, 1991), que relaciona las actitudes, las normas subjetivas y el control conductual percibida, lo que se traduce a la intención que regula el comportamiento. Se entiende actitud a las expectativas de resultados positivos o negativos de una persona hacia el comportamiento, norma subjetiva es la presión social con respecto al comportamiento, y control conductual percibido es el control percibido que tiene una persona sobre su propio comportamiento en diversas situaciones (Ajzen, 1991).

Este cuestionario y su contenido son importantes ya que logran vincular la práctica de la actividad física, el deporte y la inclusión de NNACD en el contexto educativo, tema de suma relevancia y que se propone como parte del paradigma social relacionado con el modelo ecológico de la clasificación internacional del funcionamiento (Organización Mundial de la Salud, 2001), y que a su vez se alinea con las perspectivas nacionales de Chile en el resguardo de los derechos y las oportunidades de acceso equitativo a estos espacios (Minsterio de Desarrollo Social, 2010). Otro punto importante a resaltar es que existen pocos instrumentos de este tipo, donde podemos encontrar principalmente trabajos dirigidos a la evaluación de componentes de la coordinación, el equilibrio y el desarrollo motor (Fernández-Marcote \& Navarro Leandro, 2015; Ochoa-Martínez et al., 2018; Tatar, 2018), por lo que su uso puede dar orientaciones para conocer las brechas que existen con el fin de abordarlos de modo adecuado según los lineamientos internacionales y nacionales (Organización de Naciones Unidas, 2006).

El objetivo de este estudio realizar una adecuación transcultural del cuestionario BaFSCH para el idioma español hablado en Chile verificando la exactitud de su traducción, su contenido, y su comprensión aplicado en una muestra de niñas, niños y adolescentes con discapacidad en contexto escolar.

\section{Material y Método}

Para el proceso de traducción y adaptación transcultural se siguieron los pasos metodológicos de las recomendaciones internacionales para traducción directa, síntesis de traducciones, traducción inversa, consolidación de comité de expertos y pre-test (Beaton et al., 2000; Ramada-Rodilla et al., 2013a). El proceso se detalla a continuación: 


\begin{tabular}{|c|c|c|c|c|}
\hline Cuestionario y preguntas & Versión original en Ingles & Versión traducida en Español & Ajustes para el Español & Razones de contexto \\
\hline Ítem 1. & You are a: Boy or Girl? & ¿Eres Niña o Niño? & ¿Eres niña, niño u otra opción? & $\begin{array}{l}\text { Se ajusta debido al enfoque de } \\
\text { genero/sexo. }\end{array}$ \\
\hline İtem 2. & You are in: Primary school or Secondary school? & ¿Tu estas en primaria o secundaria? & $\begin{array}{l}\text { ¿Tu estás en educación básica, } \\
\text { media, laboral u otra? }\end{array}$ & $\begin{array}{c}\text { Se ajusta según nomenclatura en } \\
\text { Chile respecto a los niveles de } \\
\text { educación. }\end{array}$ \\
\hline Ítem 3. & $\begin{array}{l}\text { Could you please tell us what kind of disability you } \\
\text { have? } \\
\text { I am missing a (part of my) arm or leg - I have trouble } \\
\text { with moving (spasticity) - I cannot see that well - I do } \\
\text { not have that much strength in my arms and/or legs - } \\
\text { Other, namely. }\end{array}$ & $\begin{array}{l}\text { ¿Que tipo de condición tienes? } \\
\text { Me falta una parte de mi cuerpo (brazo, pierna, u } \\
\text { otro) - Tengo problemas para moverme (por } \\
\text { ejemplo espasticidad) - No puedo moverme tan } \\
\text { bien - No tengo tanta fuerza para mover mis brazos, } \\
\text { piernas o mi cuerpo - otra condición. }\end{array}$ & $\begin{array}{l}\text { No puedo ver o me cuesta mucho } \\
\text { - No puedo escuchar o me cuesta } \\
\text { mucho - No logro comprender } \\
\text { bien las indicaciones. }\end{array}$ & $\begin{array}{c}\text { Se ajusta a todos los orígenes de } \\
\text { discapacidad. }\end{array}$ \\
\hline Ítem 7. & $\begin{array}{l}\text { What are those assistive devices that help you every day } \\
\text { during } \\
\text { school or playing outside? } \\
\text { Prosthesis - Wheelchair-Walker, cane } \\
\text {-White cane-Magnifying screen for the computer- } \\
\text { Speech generated devices -Splint(s)-Special shoes- } \\
\text { Adapted bicycle- Other, namely. }\end{array}$ & $\begin{array}{l}\text { ¿Ocupas alguno de estos dispositivos de ayuda? } \\
\text { Prótesis-Silla de Ruedas-Bastón-Pantalla de } \\
\text { aumento para los dispositivos-Generador por vos } \\
\text { por computadora-Ferula(s) órtesis-Zapatos } \\
\text { especiales-Bicicleta adaptada. }\end{array}$ & $\begin{array}{l}\text { Se suma: } \\
\text { Audífono o implante. }\end{array}$ & $\begin{array}{l}\text { Se ajusta a todos los orígenes de } \\
\text { discapacidad. }\end{array}$ \\
\hline
\end{tabular}

Traducción directa: se realizó una traducción conceptual del instrumento, participaron dos traductores bilingües independientes cuya lengua materna es el español. Uno de los traductores conocía el cuestionario y el otro no, posterior a su proceso entregaron los cuestionarios traducidos según lo acordado.

Síntesis de traducción: las traducciones se analizaron por ambos traductores en conjunto con el investigador principal, se identificaron, discutieron y se llego a consenso con las modificaciones. Con esto se creo el instrumento para el siguiente paso. Se hicieron algunos ajustes que se muestran en la figura 1.

Traducción inversa: la versión de síntesis fue traducida al idioma original por un tercer traductor que no conocía a los dos traductores anteriores. Se creo un informe el cual no contenía discrepancias desde el documento de síntesis.

Comité de expertos: se dispuso de un comité de 5 expertos relacionados con el área de la actividad física adaptada con una trayectoria mayor a 5 años. Se utilizó una metodología de validez de contenido según Pedrosa (2014). Después de la evaluación se hicieron los ajustes de acuerdo con la orientación de los especialistas, cuando el índice de validez de contenido (IVC) era inferior a 0,80 (Pedrosa et al., 2014). Las modificaciones se describen en la Figura 2.

\begin{tabular}{ccc}
\hline Cuestionario y preguntas & Razones de contexto & IVC \\
\hline Ítem 10 & $\begin{array}{c}\text { La educación física en Chile no aborda } \\
\text { deportes para personas con discapacidad en } \\
\text { su currículo. Se eliminan los ítems } \\
\text { mencionados para la prueba piloto. }\end{array}$ & 0,37 \\
İtem 12 & 0,21 \\
\hline Figura 2. Ajuste de modificaciones realizada en la fase de expertos &
\end{tabular}

Prueba Piloto: Para esta etapa se utilizó un proceso metodológico descriptivo de alcance transversal (Baena Paz, 2017) donde participaron NNACD de las regiones Metropolitana, Coquimbo y Valparaíso quienes asintieron su participación y en su conjunto los tutores consintieron esta. La toma de muestra fue no probabilística por conveniencia, y el proceso cuenta con la aprobación del Comité de Ética de la Universidad Santo Tomás de Santiago de Chile con código 160. 20. Los criterios de inclusión son que declaren tener alguna limitación asociada a discapacidad (de cualquier origen), y que asistan regularmente al sistema educacional en cualquier de sus niveles. Para el análisis estadístico se presentan los datos de modo descriptivo en frecuencias, porcentajes y desviaciones estándar, y se utilizó para el tratamiento el software GraphPad Prism ${ }^{\circledR}$ 8.2 (San Diego, CA, USA) y Excel® 2019.

\section{Resultados}

Se contó con 93 participantes con un promedio de edad de 13,95 años \pm 4,84 donde hubo 42 niñas que equivalen a un $45,16 \%$ donde la edad promedio corresponde a 14,26 años $\pm 4,62$, y 51 niños que corresponden a un $54,84 \%$ con 13,70 años \pm 5,04 en promedio de edad. La mayoría de las y los participantes asisten a educación básica $(62,36 \%)$, a su vez el mayor porcentaje corresponde a condiciones no definidas $(34,40 \%)$ seguido de quienes tiene dificultades para movilizarse $(12,90 \%)$ y quienes no comprenden bien las indicaciones $(12,90 \%)$. En cuanto al comienzo de sus condiciones el $(53,76 \%)$ lo adquirió previo al nacimiento, mientras que el $(46,23 \%)$ fue luego de nacer. La mayoría $(55,12 \%)$ se transporta en vehículos sin adaptaciones y ocupa silla de ruedas $(29,63 \%)$. Todos los datos anteriores se detallan en la Tabla 1.

El (56,98\%) practica actividad física o deporte en la escuela, y el $(62,36 \%)$ también lo hace fuera de ella. Destaca que las niñas se encuentran en menor proporción dentro de la práctica en la escuela $(45,23 \%)$ mientras que los niños corresponden a (54,76\%). La mayoría no participa de la organización de clubes deportivos $(83,87 \%)$ y su principal actividad es la educación física regular $(68,42 \%)$. Se observan los detalles en la Tabla 2. 
Tabla 1

\begin{tabular}{|c|c|c|c|}
\hline \multirow{2}{*}{ Variables } & Total & Niñas & \multirow{2}{*}{$\begin{array}{c}\text { Niños } \\
\mathrm{n}=51(54,84 \%)\end{array}$} \\
\hline & $\mathrm{n}=93(100 \%) \mathrm{n}=$ & $=42(45,16 \%)$ & \\
\hline Edad (en años) & $13,95 \pm 4,84 \quad 1$ & $14,26 \pm 4,62$ & $13,70 \pm 5,04$ \\
\hline \multicolumn{4}{|c|}{ Nivel escolar } \\
\hline Pre-básico & $4(4,30 \%)$ & $1(2,38 \%)$ & $3(5,88 \%)$ \\
\hline Básico & $58(62,36 \%)$ & $25(59,52 \%)$ & $33(64,70 \%)$ \\
\hline Medio & $19(20,43 \%)$ & $11(26,19 \%)$ & $8(15,68 \%)$ \\
\hline Laboral & $11(11,82)$ & $4(9,52 \%)$ & $7(13,72 \%)$ \\
\hline En casa & $1(1,07 \%)$ & $1(2,38 \%)$ & \\
\hline \multicolumn{4}{|c|}{ ¿Que condición tienes? } \\
\hline Me falta una parte de mi cuerpo. & $1(1,07 \%)$ & $1(2,38 \%)$ & - \\
\hline Tengo problemas para moverme (espasticidad & $4(4,30 \%)$ & $2(4,76 \%)$ & $2(3,92 \%)$ \\
\hline No puedo moverme tan bien. & $12(12,90 \%)$ & $6(14,28 \%)$ & $6(11,76 \%)$ \\
\hline No tengo tanta fuerza para mover mi cuerpo & $6(6,45 \%)$ & $5(11,90 \%)$ & $1(1,96 \%)$ \\
\hline No puedo ver o me cuesta mucho. & $21(22,58 \%)$ & $9(21,42 \%)$ & $12(23,52 \%)$ \\
\hline No puedo escuchar o me cuesta mucho. & $5(5,37 \%)$ & $3(7,14 \%)$ & $2(3,92 \%)$ \\
\hline No comprendo bien las indicaciones. & $12(12,90 \%)$ & $3(7,14 \%)$ & $9(17,64 \%)$ \\
\hline Otra condición. & $32(34,40 \%)$ & b) $13(30,95 \%)$ & $19(37,25 \%)$ \\
\hline \multicolumn{4}{|c|}{ ¿Cuándo comenzó tu condición? } \\
\hline Cuando nací o antes & $50(53,76 \%)$ & $23(54,76 \%)$ & $27(52,94 \%)$ \\
\hline Luego de nacer o ya de más grande & $43(46,23 \%)$ & $19(45,23 \%)$ & $24(47,05 \%)$ \\
\hline \multicolumn{4}{|c|}{ ¿Como vas normalmente a la escuela? } \\
\hline En transporte adaptado personal & $15(19,23 \%)$ & $5(11,90 \%)$ & $10(27,77 \%)$ \\
\hline En transporte sin adaptaciones & $43(55,12 \%)$ & $28(66,66 \%)$ & $15(41,66 \%)$ \\
\hline Me voy caminando o en silla de ruedas & $20(25,64 \%)$ & $9(21,42 \%)$ & $11(30,55 \%)$ \\
\hline \multicolumn{4}{|c|}{ ¿Ocupas algún dispositivo que te ayude a tus actividades? } \\
\hline No & $66(70,96 \%)$ & $31(73,81 \%)$ & $35(68,62 \%)$ \\
\hline $\mathrm{Si}$ & $27(29,03 \%)$ & $11(26,19 \%)$ & $16(31,37 \%)$ \\
\hline \multicolumn{4}{|c|}{ ¿Cual dispositivo de ayuda ocupas? } \\
\hline \multicolumn{4}{|c|}{ Puede responder más de una - solo quienes respondieron que si en la pregunta anterior } \\
\hline Prótesis & $1(3,70 \%)$ & $1(8,33 \%)$ & - \\
\hline Silla de Ruedas & $8(29,63 \%)$ & $4(33,33 \%)$ & $4(26,66 \%)$ \\
\hline Bastón & $4(14,81 \%)$ & $3(25,00 \%)$ & $1(6,66 \%)$ \\
\hline Pantalla de aumento para ver & $2(7,40 \%)$ & $1(8,33 \%)$ & $1(6,66 \%)$ \\
\hline Generador por voz para computadores & $3(11,11 \%)$ & - & $3(20,20 \%)$ \\
\hline Férula / órtesis & $4(14,81 \%)$ & $2(16,66 \%)$ & $2(13,33 \%)$ \\
\hline Zapatos Especiales & - & - & - \\
\hline Bicicleta Adaptada & - & - & - \\
\hline Audífono / implante & $3(11,11 \%)$ & $1(8,33 \%)$ & $2(13,33 \%)$ \\
\hline Anteojos & $1(3,70 \%)$ & - & $1(6,66 \%)$ \\
\hline Otro & $1(3,70 \%)$ & - & $1(6,66 \%)$ \\
\hline
\end{tabular}

Datos de Edad expresado en Frecuencia y Desviación Estándar \pm

Respuestas expresadas en Frecuencia y Porcentaje (\%)

Tabla 2

\begin{tabular}{|c|c|c|c|c|}
\hline Pregunta & Respuesta & Total & Niñas & Niños \\
\hline${ }_{¿}$ Practicas actividad física & $\mathrm{Si}$ & $53(56,98 \%)$ & $19(45,23 \%)$ & $34(66,66 \%)$ \\
\hline y/o deporte en la escuela? & No & $40(43,01 \%)$ & $23(54,76 \%)$ & $17(33,33 \%)$ \\
\hline ¿Practicas Actividad Física & $\mathrm{Si}$ & $58(62,36 \%)$ & $22(52,38 \%)$ & $36(70,58 \%)$ \\
\hline $\begin{array}{c}\text { y/o Deporte fuera de la } \\
\text { Escuela }\end{array}$ & No & $35(37,63 \%)$ & $20(47,61 \%)$ & $15(29,41 \%)$ \\
\hline${ }_{i}$ Eres miembro de algún & $\mathrm{Si}$ & $15(16,12 \%)$ & $7(16,66 \%)$ & $8(15,68 \%)$ \\
\hline club deportivo? & No & $78(83,87 \%)$ & $35(83,33 \%)$ & $43(84,31 \%)$ \\
\hline ¿Que Tipo de Actividad & $\begin{array}{c}\text { Educación Física } \\
\text { Regular }\end{array}$ & $52(68,42 \%)$ & $19(65,51 \%)$ & $33(70,21 \%)$ \\
\hline $\begin{array}{l}\text { Física Practicas? } \\
\text { * Puede responder más de una }\end{array}$ & $\begin{array}{c}\text { Talleres de Actividad } \\
\text { Física }\end{array}$ & $13(17,10 \%)$ & $5(17,24 \%)$ & $8(17,02 \%)$ \\
\hline & Talleres Deportivos & $11(14,47 \%)$ & $5(17,24 \%)$ & $6(12,76 \%)$ \\
\hline
\end{tabular}

Respuestas expresadas en Frecuencia y Porcentaje (\%)

Tabla 3.

Facilitadores para la práctica de la actividad fisica y / o deporte Cómo llegaste a practicar actividad física o deporte?

$*$ Puede responder más de una / Solo responden quienes practican actividad fisica o deporte.

\begin{tabular}{cccc}
\hline Total Niñas Niños & & & \\
\hline El doctor/equipo de salud me lo recomendó & $12(14,81 \%)$ & $9(29,03 \%)$ & $2(4,08 \%)$ \\
Alguien del centro de rehabilitación me comentó & $4(4,93 \%)$ & $2(6,45 \%)$ & $2(4,08 \%)$ \\
Alguien de la escuela me dijo & $17(20,98 \%)$ & $5(16,12 \%)$ & $12(24,49 \%)$ \\
Amigos me invitaron & $5(6,17 \%)$ & - & $5(10,20 \%)$ \\
Mis padres o tutores me incentivaron & $20(24,69 \%)$ & $7(22,58 \%)$ & $13(26,53 \%)$ \\
Hubo un día en la escuela pude probar deportes & $11(13,58 \%)$ & $3(9,67 \%)$ & $8(16,32 \%)$ \\
Me enteré por internet & $4(4,93 \%)$ & $1(3,22 \%)$ & $3(6,12 \%)$ \\
Por las clases de la escuela & $2(2,46 \%)$ & $1(3,22 \%)$ & $1(2,04 \%)$ \\
Por que me gusta & $2(2,46 \%)$ & $1(3,22 \%)$ & $1(2,04 \%)$ \\
Por el profesor de educación física & $4(4,93 \%)$ & $2(6,45 \%)$ & $2(4,08 \%)$ \\
\hline \multicolumn{4}{c}{ Por qué querías hacer actividad física o deporte? } \\
* Puede responder más de una / Solo responden quienes practican actividad fisica o deporte \\
\hline Porque es saludable & $1(0,76 \%)$ & $1(2,12 \%)$ \\
\hline Porque el doctor me dijo que es bueno para mi & $6(4,61 \%)$ & $6(12,76 \%)$ \\
Porque es bueno para mi & $41(31,53 \%)$ & $18(38,29 \%)$ & $23(27,71 \%)$ \\
Me gusta correr & $1(0,76 \%)$ & - & $1(1,20 \%)$ \\
Porque me gusta el deporte & $33(25,38 \%)$ & $8(17,02 \%)$ & $25(30,12 \%)$ \\
Ayuda a estar feliz & $1(0,76 \%)$ & - & $1(1,20 \%)$ \\
Me gusta bailar & $1(0,76 \%)$ & $1(2,12 \%)$ & - \\
Porque mis amigos practican & $9(6,92 \%)$ & - & $9(10,84 \%)$ \\
Porque puedo conocer otros niños & $9(6,92 \%)$ & $3(6,38 \%)$ & $6(7,22 \%)$ \\
Porque puedo adelgazar & $10(7,69 \%)$ & $5(10,63 \%)$ & $5(6,02 \%)$ \\
Porque mi familia practica & $4(3,07 \%)$ & $2(4,25 \%)$ & $2(2,41 \%)$ \\
Porque puedo mejorar mis habilidades & $3(2,30 \%)$ & $1(2,12 \%)$ & $2(2,41 \%)$ \\
Porque puedo ser más fuerte & $11(8,46 \%)$ & $2(4,25 \%)$ & $9(10,84 \%)$ \\
\hline Respuestas expresadas en Frecuencia y Porcentaje $(\%)$ & & &
\end{tabular}

Tabla 4.

Barreras para la práctica de la Actividad Física y / deporte

\begin{tabular}{|c|c|c|c|}
\hline \multicolumn{4}{|c|}{$\begin{array}{c}\text { ¿Por qué no practicas actividad física y/o deporte en la escuela? } \\
*_{\text {responden solo quienes no hacen actividad fisica en la escuela }}\end{array}$} \\
\hline Total Niñas Niños & & & \\
\hline No me gustan los deportes & $14(35,00 \%)$ & $12(52,17 \%)$ & $2(11,11 \%)$ \\
\hline $\begin{array}{l}\text { Tengo problemas para practicar deportes debido a mi } \\
\text { discapacidad. }\end{array}$ & $11(27,50 \%)$ & $5(21,73 \%)$ & $6(33,33 \%)$ \\
\hline Tengo miedo de caerme y lastimarme & $7(17,50 \%)$ & $3(13,04 \%)$ & $5(27,77 \%)$ \\
\hline $\begin{array}{l}\text { No me gusta cuando otras personas tienen que } \\
\text { ayudarme a hacer deporte }\end{array}$ & $2(5,00 \%)$ & & $2(11,11 \%)$ \\
\hline No hay niños de mi edad con quienes hacer deporte & $3(7,50 \%)$ & $2(8,69 \%)$ & $1(5,55 \%)$ \\
\hline No puedo encontrar un deporte para practicar & $3(7,50 \%)$ & $1(4,34 \%)$ & $2(11,11 \%)$ \\
\hline
\end{tabular}

Como principales facilitadores para la práctica de la actividad física y/o deporte se puede ver que los padres o tutores $(24,69 \%)$, seguido de las recomendaciones en la escuela $(20,98 \%)$ y el doctor junto al equipo de salud $(14,81 \%)$ son los principales promotores, los datos se pueden observar en la Tabla 3. Se observa que consideran como «buena para ellas y ellos» la práctica de la actividad física $(31,53 \%)$ además de una gran afinidad por el deporte $(25,38 \%)$ (Tabla 3$)$. En cuanto a las barreras de quienes no participan se indica como la principal, al contrario del grupo que si lo hace, el no gusto por el deporte $(35,00 \%)$ seguido de su discapacidad como factor relevante $(27,50 \%)$ y el miedo a tener un accidente $(17,50 \%)$ (Tabla 4).

\section{Discusión}

El objetivo de este estudio fue realizar una adecuación transcultural del cuestionario «Barriers and facilitators of sports in children with physical disabilities» al idioma español hablado en Chile.

Es muy importante considerar este tipo de procesos al momento de aplicar instrumentos para valorar diversos tipos de indicadores o variables de alguna población relativa a la actividad física, ya que de este modo se puede obtener información relevante y fidedigna que puede impactar en los lineamientos y acciones para favorecer el desarrollo de facilitadores en las comunidades desde un modelo ecológico (Hutzler, 2007) y que si se desarrolla bajo una metodología adecuada desde los procesos de la traducción y análisis cultural, da la posibilidad de poder conocer las realidades que podríamos encontrar en nuestros contextos disminuyendo así los sesgos que podemos encontrar desde la información que puede verse asociada de manera errónea. (RamadaRodilla et al., 2013b). En cuanto al proceso en particular del trabajo del cuestionario $\mathrm{BaFSCH}$, se consideraron las etapas propuestas según la metodología, por lo que creemos que el uso de este sería adecuado a Chile desde la perspectiva de la validez de su contenido que es fundamental (García deYébenes Prous et al., 2009). 
El análisis de la prueba piloto nos entrega información valiosa respecto al proceso de inclusión de NNACD en el contexto de la actividad física, esto ya que nos da una visión incipiente de cuales podrían ser algunos hallazgos relevantes con el fin de fortalecer las brechas que se muestran. Observamos que un alto porcentaje que equivale al $43,01 \%$ no participa de actividades relacionadas a las actividad física en el contexto educativo, lo que por una parte puede impactar profundamente en sus posibilidades de desarrollo integral (Reina Vaillo, Raúl \& Roldan, Alba, 2019), y que a su vez limita el derecho a la práctica de la actividad física como parte de un modelo social actual que debemos considerar sin lugar a dudas en base las políticas nacionales e internacionales (Ministerio del Deporte, 2015b; Organización de las Naciones Unidas, 2018). En este mismo sentido, se suma la importancia de observar que los principales precursores y motivadores para la práctica de la actividad física son los padre y tutores quienes pasan a ser actores clave en ese modelo (Fitzgerald, 2018), dejando de lado y al margen a profesoras y profesores de educación física, lo que se puede deber a sus escasos niveles de formación en el área y las barreas física, actitudinales y de la comunidad que se encuentran en Chile al momento de estos espacios (Muñoz Hinrichsen, Fernando, 2022). En este caso también se puede deber a que las bases curriculares se encuentran orientadas a grupos homogéneos de estudiantes con un abordaje bajo o casi nulo a las diversas condiciones que pueden aparecer en la asignatura de Educación Física. (Castillo-Retamal et al., 2021b)

Se muestra que existe una noción de bienestar y de consideración a que la actividad física y el deporte son una alternativa beneficiosa para el desarrollo integral y que se asocia a un gusto por el deporte y a entender que se considera como un espacio bueno para los participantes. Esto es muy importante, tal como se planta en las guías internacionales y recomendaciones de las organizaciones internacionales, donde en el Plan de Acción Mundial sobre Actividad física al 2030 se plantean beneficios similares a los nombrados por los participantes y se propone crear sociedades, entornos, poblaciones y sistema activos con el fin de fortalecer estos principios (Foster et al., 2018). En Chile según los indicadores de los hábitos de actividad física en personas con discapacidad, podemos observar que el $60 \%$ de la población de este colectivo es inactiva y el 20\% parcialmente inactivos (Ministerio del Deporte, 2020), por lo que es muy importante fomentar este tipo de estilos de vida a corta edad para tener comunidades saludables en línea con lo que se plantea desde las propuestas internacionales (Cristi-Montero et al., 2019)

\section{Conclusiones}

El cuestionario BaFSCH traducido se puede considerar como un instrumento adecuado para la verificación de facilitadores y barreras en niñas, niños y adolescentes con discapacidad para la inclusión en actividad y educación física en el contexto escolar en Chile, esto además bajos los argumentos basados en los derechos humanos y sociales que se resguardan en las leyes y convenciones internacionales. Es una herramienta que puede dar luces de como abordar estás temáticas y permitir una participación efectiva en espacios de relevancia social. Es importante destacar que pueden surgir dificultades en la aplicación del instrumento ya que requiere del apoyo de adultos para la respuestas de algunos participantes, lo que puede ser una dificultad para contar con el tiempo de los asistentes o apoyos. Otro punto importante es que la muestra era seleccionado por conveniencia, por lo que se recomienda que futuras investigaciones puedan abordar grupos de mayor tamaño muestral con el fin de conocer de modo más acabado este fenómeno. Se propone poder relacionar este estudio con los hábitos de la práctica de la actividad física y las recomendaciones internacionales en este ámbito planteada por los organismos competentes como la Organización Mundial de la Salud o el Ministerio de Salud de Chile.

\section{Referencias}

Ajzen, I. (1991). The theory of planned behavior. Organizational Behavior and Human Decision Processes, 50(2), 179-211. https://doi.org/10.1016/07495978(91)90020-T

Baena Paz, G. (2017). Metodología de la investigación (3a. Ed.). Grupo Editorial Patria.

Beaton, D. E., Bombardier, C., Guillemin, F., \& Ferraz, M. B. (2000). Guidelines for the Process of CrossCultural Adaptation of Self-Report Measures: Spine, 25(24), 3186-3191. https://doi.org/10.1097/ 00007632-200012150-00014

Castillo-Retamal, F., Cárcamo Garrido, B., Aravena Calderón, H., Valenzuela Zakuda, A., Pérez Farías, T., Medel Tapia, C., \& Quezada Alacaino, J. (2021a). Necesidades Educativas Especiales y Educación Física: Un análisis desde la propuesta curricular ministerial de Chile (Special Educational Needs and 
Physical Education: an analysis from the Chilean ministerial curricular proposal). Retos, 42, 56-65. https://doi.org/10.47197/retos.v42i0.86977

Castillo-Retamal, F., Cárcamo Garrido, B., Aravena Calderón, H., Valenzuela Zakuda, A., Pérez Farías, T., MedelTapia, C., \& Quezada Alacaino, J. (2021b). Necesidades Educativas Especiales y Educación Física: Un análisis desde la propuesta curricular ministerial de Chile (Special Educational Needs and Physical Education: an analysis from the Chilean ministerial curricular proposal). Retos, 42, 56-65. https: / / doi.org/10.47197/retos.v42i0.86977

Cordente-Mesas, D., González-Víllora, S., Block, M. E., \& Contreras-Jordán, O. R. (2016). Structure, validity and reliability of the Children's Attitudes Towards Integrated Physical Education-Spanish version (CAIPE-SP). 9(2), 3-12. https://doi.org/10.5507/euj.2016.005

Cristi-Montero, C., Sadarangani, K. P., GarridoMéndez,A., Poblete-Valderrama, F., Díaz-Martínez, X., \& Celis-Morales, C. (2019). Relación entre niveles de actividad física y sedentarismo con síndrome metabólico. ENS Chile 2009-2010. Salud Pública de México, 61(2, Mar-Abr), 166. https://doi.org/ $10.21149 / 8879$

Fernández-Marcote, A. E., \& Navarro Leandro, A. (2015). Análisis comparativo de la coordinación óculo-segmentaria en tres grupos de población: Síndrome de down, Deficiencia mental y sin discapacidad. Retos, 1, 17-20. https://doi.org/ 10.47197/retos.v0i1.35107

Fitzgerald, H. (2018). Disability and Barriers to Inclusion. En I. Brittain \& A. Beacom (Eds.), The Palgrave Handbook of Paralympic Studies (pp. 55-70). Palgrave Macmillan UK. https: / / doi.org/10.1057/ 978-1-137-47901-3_4

Fondo de las Naciones Unidas para la Infancia. (1989). Conveción de los Derechos de los Niños. https:// www.unicef.org/chile/media/3176/file/ convencion_sobre_los_derechos_del_nino.pdf

Foster, C., Shilton, T., Westerman, L., Varney, J., \& Bull, F. (2018). World Health Organisation to develop global action plan to promote physical activity: Time for action. British Journal of Sports Medicine, 52(8), 484485. https://doi.org/10.1136/bjsports-2017098070

García de Yébenes Prous, M. J., Rodríguez Salvanés, F., \& Carmona Ortells, L. (2009). Validación de cuestionarios. Reumatología Clínica, 5(4), 171-177. https:/ /doi.org/10.1016/j.reuma.2008.09.007

Hutzler, Y. (2007). A Systematic Ecological Model for
Adapting Physical Activities: Theoretical Foundations and Practical Examples. Adapted Physical Activity Quarterly, 24(4), 287-304.https://doi.org/10.1123/ apaq.24.4.287

Hutzler, Y., \& Sherrill, C. (2007). Defining Adapted Physical Activity: International Perspectives. Adapted Physical Activity Quarterly, 24(1), 1-20. https:// doi.org/10.1123/apaq.24.1.1

Jaarsma, E. A., Dijkstra, P. U., de Blécourt, A. C. E., Geertzen, J. H. B., \& Dekker, R. (2015). Barriers and facilitators of sports in children with physical disabilities: A mixed-method study. Disability and Rehabilitation, 37(18), 1617-1625. https://doi.org/ 10.3109/09638288.2014.972587

Jaarsma, E. A., Geertzen, J. H. B., de Jong, R., Dijkstra, P. U., \& Dekker, R. (2014). Barriers and facilitators of sports in Dutch Paralympic athletes: An explorative study: Barriers and facilitators of sports. Scandinavian Journal of Medicine \& Science in Sports, 24(5), 830-836. https://doi.org/10.1111/ sms. 12071

Ministerio de Educación. (2015). Ley 20.845 que reconoce la Educación Inclusiva. Chile. https://www.bcn.cl/ leychile/navegar?idNorma $=1078172$

Ministerio del Deporte. (2015a). Ley 20.978 que reconoce el Deporte Adaptado y Paralímpico. Chile. https:// www.bcn.cl/leychile/navegar?idNorma $=1098002$

Ministerio del Deporte. (2015b). Política Nacioanal de la Actividad Física y el Deporte 2015-2025. https:// biblioteca.digital.gob.cl/handle/123456789/380

Ministerio del Deporte. (2020). II Estudio Nacional de hábitos de actividad física y deporte en población con discapacidad en Chile. https://mindep.cl/secciones/ 153

Ministerio de Desarrollo Social. (2010). Ley 20.422 que estable las normas sobre la igualdad de oportunidades e inclusión social de personas con discapacidad. Chile. ht tp s : / / www.bcn.cl/leychile / navegar?idLey $=20422$

Muñoz Hinrichsen, F. I., Bossay Salinas, C., Henriquez Valenzuela, M., Martinez Aros, A., \& Castelli Correira Campos, L. F. (2020). Deporte paralímpico en Chile, una aproximación a la realidad nacional en el año 2019. Revista Iberoamericana de Ciencias de la Actividad Física y el Deporte, 9(3), 91-101. https:// doi.org/10.24310/riccafd.2020.v9i3.8575

Muñoz Hinrichsen, Fernando, M. (2022). Formación del profesorado de educación física vinculado a la inclusión y la discapacidad en universidades de Chile. Journal of Movement and Healt, 19(1). https://doi.org/ 
10.5027/jmh-Vol19-Issue1(2022)art134

Ocete calvo, C., Pérez-Tejero, J., Franco, E., \& Coterón, J. (2017). Validación de la versión española del cuestionario «Actitudes de los alumnos hacia la integración en educación física (CAIPE-R)». Psychology, Society \& Education, 9(3), 447. https:/ / doi.org/10.25115/psye.v9i3.1025

Ochoa-Martínez, P. Y., Hall López, J. A., Carmona López, A. A., Reyes Castro, Z. E., Sáenz-López Buñuel, P., \& Conde García, C. (2018). Análisis comparativo de un programa educación física en niños con discapacidad auditiva sobre la edad motora equivalente (Comparative analysis of the effect of physical education program of motor age equivalent in children with hearing disability). Retos, 35, 310-313. https: / / doi.org/10.47197/retos.v0i35.67190

Organización de las Naciones Unidas. (2018). Educación 2030, Declaración de Incheon y Marco de Acción para la realización del Objetivo de Desarrollo Sostenible 4. https: / /www.gcedclearinghouse.org/sites/default/files/ resources/245656s.pdf

Organización de Naciones Unidades para la Educación, la Ciencia y la Cultura. (2015). Carta internacional de la educación física, la actividad física y el deporte. https: / / unesdoc.unesco.org/ark: / 48223 / pf0000235409_spa

Organización de Naciones Unidas. (2006). Convención de los Derechos sobre la Personas con Discapacidad. https: / /www.un.org/esa/socdev/ enable/documents / tccconvs.pdf

Organización de Naciones Unidas. (2015). Transformando Nuestro Mundo: Agenda al 2030 de los Objetivos del Desarrollo Sostenible. https: / /sdgs.un.org/2030agenda Organización Mundial de la Salud. (2001). Clasificación Internacional del Funcionamiento, la Salud y la
Discapacidad. https: / /www. who.int/standards / classifications / international-classification-offunctioning-disability-and-health

Pedrosa, I., Suárez-Álvarez, J., \& García-Cueto, E. (2014). Evidencias sobre la Validez de Contenido: Avances Teóricos y Métodos para su Estimación [Content Validity Evidences: Theoretical Advances and Estimation Methods]. Acción Psicológica, 10(2), 3. https: / / doi.org/10.5944/ap.10.2.11820

Ramada-Rodilla, J. M., Serra-Pujadas, C., \& DelclósClanchet, G. L. (2013a). Adaptación cultural y validación de cuestionarios de salud: Revisióny recomendaciones metodológicas. 55(1), 57-63.

Ramada-Rodilla, J. M., Serra-Pujadas, C., \& DelclósClanchet, G. L. (2013b). Adaptación cultural y validación de cuestionarios de salud: Revisióny recomendaciones metodológicas (N. $\left.{ }^{\circ} 1\right) .55(1), 57-63$.

Reina, R., Hutzler, Y., Iniguez-Santiago, M. C., \& Moreno-Murcia, J. A. (2016). Attitudes towards Inclusion of Students with Disabilities in Physical Education Questionnaire (AISDPE):A two-component scale in Spanish. 36, 75-87.

Reina Vaillo, Raúl, \& Roldan, Alba. (2019). ACTIVIDAD FISICA ADAPTADA PARA PERSONAS EN SITUACION DE DISCAPACIDAD (Muñoz Hinrichsen, Fernando, Ed.). RIL EDITORES.

Tamayo, M., Besoaín, Á., \& Rebolledo, J. (2018). Determinantes sociales de la salud y discapacidad: Actualizando el modelo de determinación. Gaceta Sanitaria, 32(1), 96-100. https://doi.org/10.1016/ j.gaceta.2016.12.004

Tatar, Y. (2018). Applicability of Standardized Physical Fitness Tests in Children with Different Types of Disabilities. International Journal of Special Education, 33, 687-703.

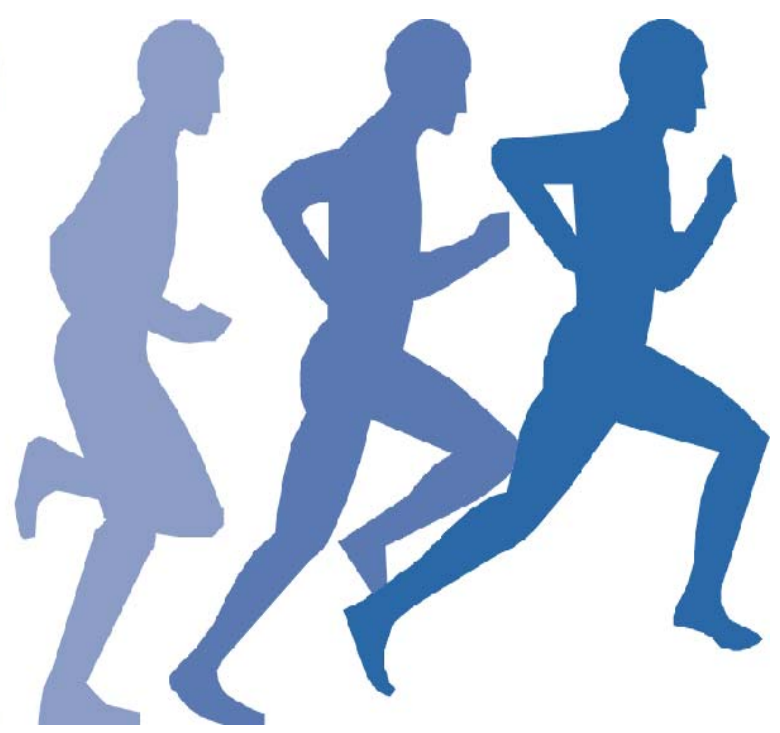

